\title{
BIM NA AVALIAÇÃO DO CICLO DE VIDA DE EDIFICAÇÕES: REVISÃO DA LITERATURA E ESTUDO COMPARATIVO
}

\section{BUILDING INFORMATION MODELLING (BIM) IN LIFE CYCLE ASSESMENT: LITERATURE REVIEW AND COMPARATIVE STUDY}

\author{
Natalia Nakamura Barros ${ }^{1}$ \\ Universidade Estadual de Campinas, Campinas, SP, Brasil, natalia.nakamura@ fec.unicamp.br \\ Vanessa Gomes da Silva ${ }^{2}$ \\ Universidade Estadual de Campinas, Campinas, SP, Brasil, vangomes@fec.unicamp.br
}

\section{Resumo}

A necessidade da redução do consumo de energia elétrica e de emissão de GHGs (GreenHouse Gases) já é consensual. Sabe-se que edifícios consomem quantidades significativas de energia, além de emitirem grandes quantidades de GHGs. Devido a essa enorme influência sobre a sociedade, é fundamental para arquitetura e construção estar consciente do seu impacto, e desenvolver ferramentas eficazes que são capazes de medir a origem e a possível redução destes impactos, com o uso da avaliação do ciclo de vida de edificações (ACV) aliada ao Building Information Modeling (BIM). Este artigo fornece uma contribuição teórica no campo de integração de BIM e avaliação de ciclo de vida (ACV). A revisão de literatura evidencia que as publicações no tema BIM/ACV concentram-se no desenvolvimento e no melhoramento das ferramentas computacionais para auxiliar a ACV nas fases de projeto e de construção de edificações. São poucas as contribuições que tratam do processo de trabalho. Para contribuir no preenchimento desta lacuna de conhecimento, este artigo analisa o impacto potencial trazido pela adoção de BIM na ACV da perspectiva de processo, tecnologia associada e informação trocada. O método compõe-se de uma revisão sistemática de literatura (RSL), seguida da análise de fluxos de trabalho de avaliação tradicional de energia e emissão de GHGs incorporadas no ciclo de vida comparativamente ao fluxo de trabalho mediado por BIM. A RSL identificou lacunas e tendências de pesquisa, e embasou a elaboração de fluxos de trabalho analisados. Da análise comparativa depreendeu-se que a adoção de BIM no fluxo de trabalho de avaliação de energia incorporada e emissão de GHGs no ciclo de vida de edificações, tem impacto variado no processo de projeto e alto impacto na coleta de dados. A principal vantagem do uso de BIM na ACV é a sua capacidade de otimizar o processo, auxiliar a tomada de decisão durante toda a evolução do projeto e instigar a convergência para uma solução otimizada.

Palavras-chave: BIM. Energia incorporada. Emissão de GHG. Avaliação do ciclo de vida.

\begin{abstract}
The need to reduce energy consumption and emissions of greenhouse gases (GHG) has come to the forefront of international discussion and efforts. In this sense, combining Life Cycle Assessment (LCA) with Building Information Modeling (BIM) constitutes a field full of potential that however remains underexplored. A literature review showed that most papers published on the intersection between BIM and LCA, have focused on the development and improvement of computational tools to assist LCA in building design and construction. Few contributions are available on the workflow. This paper, in addition to presenting the review of the literature conducted, investigates the potential impact brought by the adoption of BIM in LCA emphasizing the process, the associated technology and information exchanged. The method encompasses a systematic literature review, followed by a comparative analysis of traditional manual information input versus BIM-aided lifecycle assessment. This paper provides a theoretical contribution on BIM and lifecycle assessment integration. The systematic literature review identified recent research trends and gaps, and launched the base for developing the workflows examined. The analysis showed that adoption of BIM-aided lifecycle LCee / LCeghg have a variable impact in design process and a high impact in data collection phase. The most distinctive advantage of BIM-aided LCA is its capacity for process optimization, the possibility to iteratively support design decision-making instigating convergence to an optimal solution.
\end{abstract}

Keywords: BIM. Embodied Energy. GHG Emission. Life cycle assessment.

\section{How to cite this article:}

BARROS, Natalia Nakamura; SILVA, Vanessa Gomes da. Bim na avaliação do ciclo de vida de edificações: revisão da literatura e estudo comparativo. PARC Pesquisa em Arquitetura e Construção, Campinas, SP, v. 7, n. 2, p. 89-101, jun. 2016. ISSN 1980-6809. Disponível em: <http://periodicos.sbu.unicamp.br/ojs/index.php/parc/article/view/8645401>. Acesso em: 30 jun. 2016. doi:http://dx.doi.org/10.20396/parc.v7i2.8645401. 


\section{Introdução}

A Avaliação do Ciclo de Vida (ACV) é uma compilação e avaliação das entradas, das saídas e dos impactos ambientais potenciais de um sistema de produto ao longo do seu ciclo de vida. No Brasil, a ACV foi normatizada pela NBR ISO 14040: gestão ambiental, avaliação do ciclo de vida - princípios e estrutura e NBR ISO 14044: gestão ambiental, avaliação do ciclo de vida - requisitos e orientações (ASSOCIAÇÃO BRASILEIRA DE NORMAS TÉCNICAS, 2009).

A quantificação dos impactos ambientais do projeto para ACV é dificultada principalmente devido à falta de informações sobre os materiais que são armazenados no banco de dados, e por falhas de interoperabilidade entre as ferramentas de projeto e ferramentas que permitem as avaliações do ciclo de vida completo das edificações (BASBAGILL et al., 2013; DIAZ; ANTON, 2014; JRADE; JALAEI, 2013). Além disso, um dos maiores problemas da ACV convencional é o longo tempo consumido (DIAZ; ANTON, 2014).

A NBR ISO 14044 divide a ACV em quatro fases, sendo estas: definição do objetivo e escopo, análise de inventário, avaliação de impacto e interpretação (ASSOCIAÇÃO BRASILEIRA DE NORMAS TÉCNICAS, 2009). A etapa de análise de inventário consiste na identificação e quantificação dos produtos da edificação. Já a avaliação de impacto consiste no cálculo dos indicadores ambientais analisados de acordo com a metodologia adotada.

O cálculo de emissões GHGs incorporadas consiste no somatório das emissões, em $\mathrm{kgCO} 2_{\mathrm{eq}}$ incorporadas (nos produtos e atividades de construção, na manutenção e no fim de vida) multiplicadas pelos fatores de emissão equivalente (IPCC, 2007). A contribuição total da edificação em seu ciclo de vida completo resulta da soma destas emissões incorporadas, com as emissões associadas ao uso de energia na operação e demais atividades de manutenção da edificação ao longo de sua vida útil. Analogamente, o cálculo de energia incorporada consiste no somatório do uso de diferentes recursos energéticos em cada fase do ciclo de vida (exceto a energia operacional, considerada à parte), multiplicadas pelos fatores de caracterização correspondentes. $\mathrm{O}$ emprego de plataformas de apoio a ACV, facilita consideravelmente estes cálculos, ao incorporar métodos de avaliação de impactos como o Cumulative Energy Demand - CED e o CML 2001 (SILVA et al, 2014).

Na prática típica de projeto, as avaliações de desempenho ambiental ocorrem só depois que o projeto está finalizado, dificultando possíveis alterações e melhorias. $\mathrm{O}$ uso da simulação na prática projetual requer a inserção de novas variáveis e re-simulações cíclicas sempre que uma solução inicial satisfatória não é alcançada (KOWALTOWSKI et al., 2006).

Os impactos ambientais de uma edificação são determinados principalmente a partir de decisões tomadas nos estágios iniciais de projeto (BASBAGILL et al., 2013; DIAZ; ANTON, 2014; KULAHCIOGLU et al., 2012). No entanto, as ferramentas de avaliação são usadas depois que o projeto da edificação está completo e, portanto, as decisões corretivas são caras e difíceis de implementar (DAWOOD et al., 2009).

Dada a riqueza da informação sobre os objetos necessária para executar análises energéticas e outras análises ambientais (EASTMAN et al., 2014), a modelagem de informação de edificações (BIM) pode fornecer várias vantagens. Succar (2009, p.357) define BIM como "[...] um conjunto inter-relacionado de políticas, processo e tecnologias". Esta pesquisa insere-se no campo do processo, no subcampo de atividades e fluxos de trabalho.

Quando adequadamente implementada, a modelagem de informação oferece benefícios em todas as fases do ciclo de vida de um empreendimento de construção (BECERIKGERBER; KENSEK, 2010), pois contém a geometria exata e dados relevantes para dar suporte à construção, à fabricação e ao fornecimento de insumos necessários para a realização da construção (EASTMAN et al., 2014). A execução do projeto requer planejamento detalhado e modificações fundamentais no processo de projeto dos membros da equipe para alcançar com êxito o valor a partir da informação disponível no modelo (CIC, 2011).

O objetivo do presente artigo é contribuir para os estudos de integração de BIM e ACV ao verificar o possível impacto da adoção de BIM no processo de avaliação da energia incorporada e emissão de GHGs no ciclo de vida de edificações, neste caso inscrito no limite de sistema do berço ao fim-de-vida (cradle to end of life) da edificação.

\section{Método}

Para tanto, a pesquisa foi realizada em duas etapas (Figura 1). Inicialmente, uma revisão sistemática da literatura permitiu identificar, avaliar e interpretar os estudos atuais e relevantes na interseção dos campos de BIM e ACV; posicionar esta contribuição e auxiliar no desenvolvimento dos fluxos de trabalho de ACV estudados. Concluída a revisão da literatura, procedeu-se a segunda etapa da pesquisa, centrada na pesquisa comparativa entre fluxos de trabalho de ACV com e sem auxílio de BIM, tomando como objeto um processo de planejamento de execução de projeto BIM para avaliação do consumo de energia incorporada e emissão de GHGs do berço ao fim-de-vida de uma edificação. 
Figura 1 - Esquema do método utilizado na pesquisa

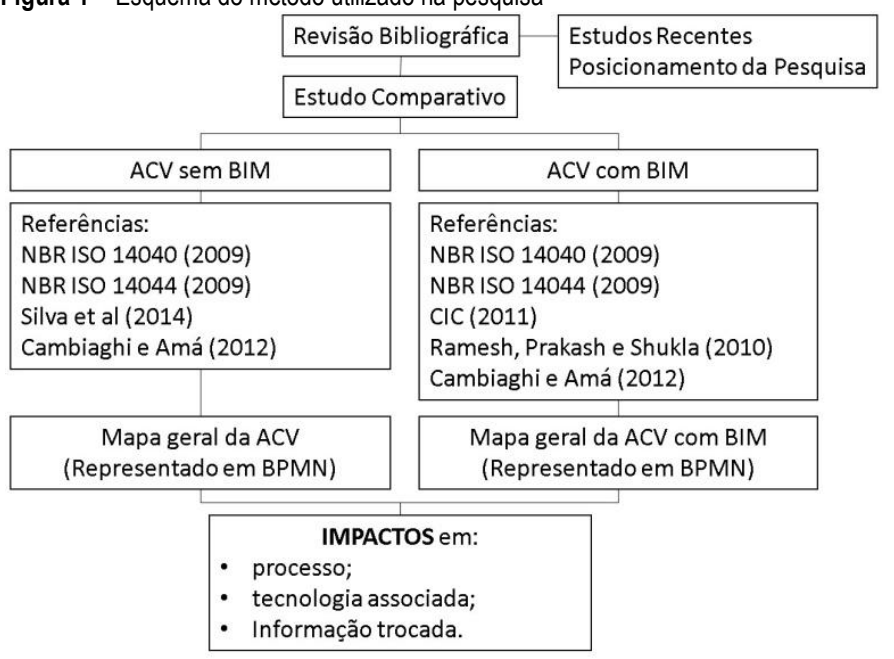

Fonte: $\mathrm{O}$ autor

A comparação entre os mapas de processo de ACV e de ACV com adoção de BIM, buscou revelar em que pontos ocorrerão mudanças na adoção de BIM no processo de avaliação de energia incorporada e emissão de GHGS do ciclo de vida de edificações, no que tange às atividades desenvolvidas no processo, à tecnologia associada e às informações trocadas.

\section{Revisão sistemática da literatura}

A revisão sistemática da literatura, proposta por Kitchenham e Charters (2007), é uma forma de estudo secundário que utiliza um protocolo bem-definido para identificar, analisar, e interpretar todas as evidências disponíveis relacionadas a uma questão de pesquisa definida, de forma que seja livre de tendências e, na extensão possível, reprodutível.

A estratégia de pesquisa adotada na revisão sistemática da literatura realizada foi a busca em bases de dados digitais relevantes na área de Arquitetura, Engenharia e Construção (AEC), sendo estas: Engineering Village, Web of Science, Avery, ASCE Library e Scopus, de artigos contendo as palavras-chave BIM e LCA (Life Cycle Assessment) presentes em todo o artigo, até o ano de 2015, conforme mostra o Quadro 1.

A partir desta pesquisa inicial obtiveram-se 116 artigos. Dentre estes, 51 artigos foram excluídos por se tratarem de duplicidade na busca por diferentes bases de dados ou pela impossibilidade de acesso ao mesmo, resultando em 65 artigos. Depois, uma análise mais aprimorada foi realizada através de uma estratégia de extração de dados, onde os seguintes dados foram extraídos de cada artigo: Autor, Ano, Objetivo, Método, Desenvolvimento, Resultados, Conclusão e Considerações Finais. A partir desta análise mais específica verificou-se que alguns artigos não abordavam os temas ACV e BIM conjuntamente, abordando apenas um dos temas com relevância, o que provocou a exclusão destes.

\begin{tabular}{|c|c|c|c|c|c|}
\hline Base de Dados Digitais & ASCE Library & Engineering Village & Scopus & Web of Science & Avery \\
\hline Estratégia de busca & $\begin{array}{l}\text { BIM in Anywhere and } \\
\text { LCA in Anywhere }\end{array}$ & $\begin{array}{l}\text { BIM in All Fields and } \\
\text { LCA in All Fields }\end{array}$ & $\begin{array}{l}\text { BIM in Article Title, } \\
\text { Abstract, Keywords e } \\
\text { LCA in Article Title, } \\
\text { Abstract, Keyword }\end{array}$ & $\begin{array}{l}\text { BIM em Tópico e LCA } \\
\text { em Tópico (Tópico } \\
\text { compreende os } \\
\text { seguintes campos: } \\
\text { Título Resumo, } \\
\text { Palavras-chave de } \\
\text { autor, Palavras- } \\
\text { chave) }\end{array}$ & $\begin{array}{l}\text { BIM em Qualquer Lugar e } \\
\text { LCA em Qualquer Lugar }\end{array}$ \\
\hline Data da Pesquisa & \multicolumn{5}{|c|}{$24 / 05 / 2016$} \\
\hline Anos cobertos pela pesquisa & \multicolumn{5}{|c|}{ Todos } \\
\hline Critérios de Inclusão & \multicolumn{5}{|c|}{ Nenhum } \\
\hline Critérios de Exclusão & $\begin{array}{l}\text { Tipo de Artigo: Front } \\
\text { Matter (Páginas de } \\
\text { matéria que vêm } \\
\text { antes dos trabalhos } \\
\text { ou capítulos em um } \\
\text { trabalho publicado) e } \\
\text { Back Matter (Páginas } \\
\text { de matéria que vêm } \\
\text { após os papéis ou } \\
\text { capítulos em um } \\
\text { trabalho publicado.) }\end{array}$ & Nenhum & Nenhum & Nenhum & Nenhum \\
\hline $\mathrm{N}^{0}$ de Artigos Encontrados & 29 & 31 & 36 & 19 & 1 \\
\hline Artigos Duplicados & \multirow{2}{*}{\multicolumn{5}{|c|}{$\begin{array}{l}51 \\
65\end{array}$}} \\
\hline Total & & & & & \\
\hline
\end{tabular}

Fonte: Barros (2016, p.111) 


\section{Estudo comparativo}

A pesquisa comparativa entre fluxos de trabalho de ACV com e sem auxílio de BIM, tomou como base o desenvolvimento de um processo de planejamento de execução de projeto BIM para avaliação do consumo de energia incorporada e emissão de GHG do berço ao fimde-vida da edificação (cradle to end of life). A revisão da literatura embasou o desenvolvimento dos fluxos de trabalho, apresentados através da notação BPMN (Business Process Model and Notation). O BPMN consiste em uma notação gráfica desenvolvida pelo Object Management Group (OMG) que tem como objetivo fornecer uma notação gráfica padrão e compreensível por todos os agentes interessados (OBJECT MANAGEMENT GROUP, 2011).

O mapa geral do processo da ACV foi desenhado de acordo com o procedimento realizado por Silva et al (2014), adaptado para o foco na avaliação do consumo de energia incorporada e emissão de GHG do berço ao fimde-vida (cradle to end of life). Com base nas etapas do processo de projeto proposto por Cambiaghi e Ama (2012), incluíram-se como participantes os seguintes agentes: projetista de arquitetura, estrutural, instalações elétricas, instalações hidro sanitárias, fundações e outros projetistas, além da diretoria da empresa, que é o participante para qual o projeto se destina, e o analista $\mathrm{ACV}$. No processo de projeto enfatizou-se a fase de projeto executivo da obra, uma vez que para a execução da ACV é necessário que o projeto da edificação esteja nesta fase a fim de que todas as informações necessárias para a ACV estejam detalhadas no projeto. Já os procedimentos da ACV foram seguidos conforme as normas NBR ISO 14040 e NBR ISO 14044 (ASSOCIAÇÃO BRASILEIRA DE NORMAS TÉCNICAS, 2009a, 2009b).

Para o desenho do mapa geral do processo em ACV com adoção de BIM são necessários: definição de usos potenciais de BIM, arranjo dos usos de BIM de acordo com o processo de projeto, identificação das partes responsáveis por cada processo, e determinação do intercâmbio de informações requerido para implementação de cada uso de BIM (CIC, 2011). O uso de BIM definido para o estudo é a análise do consumo de energia incorporada e emissão de GHGs do berço ao fimde-vida (cradle to end of life). As partes responsáveis por cada processo serão definidas de acordo com Cambiaghi e Ama (2012), e o intercâmbio de informações necessárias serão definidas de acordo com as NBR ISO 14040 e NBR ISO 14044 (ASSOCIAÇÃO BRASILEIRA DE NORMAS TÉCNICAS, 2009a, 2009b) e da tabela proposta por Ramesh, Prakash e Shukla (2010), que explicita as fontes dos dados necessários para a realização de uma ACV. Salienta-se que para este desenho utilizouse como método de integração entre ACV e BIM, a extração automática de quantitativos a partir do modelo BIM, apontada pela revisão sistemática da literatura como o método mais utilizado por pesquisadores.

\section{Análise do impacto da adoção de BIM na ACV}

A verificação dos impactos potenciais de BIM na $\mathrm{ACV}$ foi adaptada das escalas de implantação de BIM propostas por Moreira e Ruschel (2015), em relação ao processo, à tecnologia associada e à informação trocada, apresentadas no Quadro 2.

Os impactos obtidos no processo podem ser considerados: (i) de baixo impacto, quando com a adoção de BIM, o conjunto de atividades não sofre modificações, (ii) de médio impacto, quando a adoção de BIM implica no rearranjo e adição ou eliminação de atividades, e (iii) de alto impacto, quando a organização não tem explicitado o mapa de processo e este deve então ser desenhado incorporando BIM.

Inferem-se também três possíveis impactos nos softwares utilizados no procedimento de BIM+ACV: (i) de baixo impacto, quando não há alteração nos softwares utilizados; (ii) de médio impacto, quando se utiliza um software e este é substituído por BIM e (iii) de alto impacto, quando não se utiliza softwares e passa-se então a utilizar.

Da mesma forma, inferem-se três possíveis transformações da informação trocada: (i) de baixo impacto, quando a informação já é digital; (ii) de médio impacto, quando a informação era parcialmente analógica/digital e passa a ser totalmente digital; e (iii) de alto impacto quando a informação era totalmente analógica no processo tradicional, e passa a ser digital no processo com BIM.

A partir das transformações percebidas no processo, na tecnologia associada e na informação trocada, foi possível vislumbrar escalas de adoção de BIM na ACV através da soma total de impactos, variando como: baixo (3 a 4), médio (5 a 6 ) e alto ( 8 a 9).

\section{Identificação e caracterização de estudos recentes sobre integração de BIM e ACV}

A revisão sistemática de literatura revelou que existe um grande crescimento do número de publicações sobre o tema (Figura 2), evidenciando que há um interesse cada vez maior pelo tema em questão, principalmente por se tratar da união de duas áreas em desenvolvimento: tecnologia e sustentabilidade.

Muitos estudos apontam que BIM pode vir a ser um sistema importante para o desenvolvimento de projetos verdes (WU; XIA; WANG, 2015; ALWAN; JONES, 2014). Os artigos encontrados abordam principalmente a integração entre softwares BIM e ferramentas de análises ambientais. O software BIM mais utilizado foi o Autodesk 
Revit, dentre as ferramentas ACV mais utilizadas, estão o SimaPro e GABI, com foco principalmente nos indicadores de consumo de energia e emissão GHGs. As análises energéticas foram realizadas principalmente por programas como: EnergyPlus e Ecotect, e o sistema de certificação LEED também foi um assunto muito abordado nos artigos.

Os artigos demonstraram como as funcionalidades de BIM existentes podem ser adaptadas para a condução da ACV, mas grande parte delas requerem alguma conversão e entrada manual de dados. A abordagem mais pesquisada de integração foi a extração automática de dados diretamente do modelo BIM para dar à suporte $\mathrm{ACV}$, a fim de comparar alternativas nas fases iniciais de projeto, e verificaram que esta é possível (DIAZ; ANTON, 2014; IDDON; FIRTH, 2013; ALWAN; JONES, 2014; PENG,
2016; SHADRAM et al., 2015). Porém, os dados obtidos a partir desta extração não foram validados por especialistas, e, portanto, não há previsão da sua confiabilidade.

\section{BIM no auxílio às análises ambientais}

Estudos sobre a aplicabilidade de BIM no suporte aos projetos que visam à performance ambiental têm se aprimorado continuamente. Em 2014, Diaz e Anton (2014) propuseram duas possíveis abordagens para integrar BIM e ACV: 1) através da extração automática de dados a partir do modelo BIM para cálculo de ACV e 2) a partir da inclusão de indicadores ambientais em objetos BIM.

Quadro 2 - Escalas de impacto de implantação de BIM no processo de projeto de edificação com alto desempenho ambiental

\begin{tabular}{|c|c|c|c|c|}
\hline Processo & Impacto & Software & Impacto & Informação \\
\hline $\begin{array}{c}\text { Atividades não } \\
\text { sofrem alterações }\end{array}$ & 1 & $\begin{array}{c}\text { Não sofrem } \\
\text { alterações }\end{array}$ & 1 & Era digital \\
\hline $\begin{array}{c}\text { Atividades são } \\
\text { alteradas }\end{array}$ & 2 & $\begin{array}{c}\text { Usa softwares e } \\
\text { estes sofrem } \\
\text { alterações }\end{array}$ & 2 & $\begin{array}{c}\text { Era parcialmente } \\
\text { analógica/digital }\end{array}$ \\
\hline $\begin{array}{c}\text { Não tem atividades } \\
\text { delimitadas }\end{array}$ & 3 & $\begin{array}{c}\text { Não usa softwares } \\
\text { Impacto Total (soma): Baixo (3 a 4), Médio (5 a 6), Alto (8 a 9) }\end{array}$ & Era analógica \\
\hline
\end{tabular}

Fonte: Barros (2016, p.79)

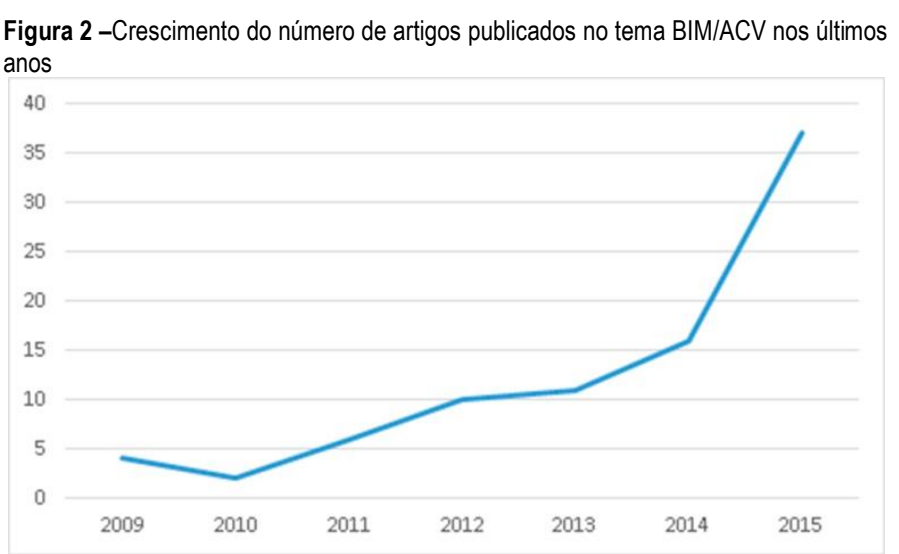

Fonte: $\operatorname{Barros}(2016$, p.60)

Iddon e Firth (2013) e Peng (2016) utilizaram o método de extração automática de quantitativos de materiais a partir do modelo BIM, para estimar emissões de carbono em edificações. Já Alwan e Jones (2014), Shadram et al. (2015) e Al-Ghamdi e Bilec (2014) utilizaram o mesmo método e incluíram também em suas análises, o consumo de energia. Os autores verificaram que o procedimento adotado permitiu simplificar a estimativa das emissões de carbono ao longo ciclo de vida de um edifício e poderia ser aplicado em outros tipos de construções (ALWAN; JONES, 2014), porém o modelo BIM não pôde fornecer dados suficientes nas fases iniciais para uso na $\mathrm{ACV}$ (PENG, 2016).
Jrade e Jalaei (2013) e Jalaei e Jrade (2014) propuseram a inclusão de informações ambientais nos objetos BIM para oferecer suporte à decisão de escolha de materiais do projetista durante a etapa de projeto da edificação. Este procedimento é realizado após a extração dos quantitativos de materiais a partir do modelo BIM e obtenção dos resultados em softwares de análises ambientais. Entretanto, verificaram que o modelo proposto não é totalmente automatizado, pois os usuários ainda têm que fazer as etapas de inserção manualmente (JRADE; JALAEI, 2013; JALAEI; JRADE, 2014).

$\mathrm{O}$ método de inserção de indicadores ambientais em objetos BIM exige tempo e esforço. No entanto, uma vez que o objeto já inclui informações ambientais, ele pode ser utilizado sempre que for necessário, sem repetir a tarefa demorada de realizar a avaliação ambiental. Por outro lado, devido ao fato de cada projeto de construção ser único e ter a sua própria localização, a informação de distância do fabricante para o local de construção seria variável, o que impossibilitaria a sua inclusão como uma informação padrão. Portanto, algumas suposições têm de ser feitas, o que significa que a precisão da avaliação ainda é reduzida (ANTON; DÍAZ).

\section{Resultados}

Desenho comparativo de fluxos de trabalho 
A Figura 3 apresenta o fluxo de trabalho de um processo de projeto de avaliação de energia e emissões $G H G$ incorporadas no ciclo de vida da edificação. Num primeiro momento, o cliente solicita o projeto ao projetista, que geralmente é o arquiteto, passando os requisitos necessários para o seu desenvolvimento (1). Após o desenvolvimento do projeto arquitetônico 2D, este é passado para os engenheiros civil, elétrico, hidráulico e outros projetistas (2), para que cada um elabore o seu respectivo projeto (CAMBIAGHI; AMA, 2012). Após a análise de compatibilidade entre projetos, o projeto executivo é então passado ao especialista ACV que realiza a análise a partir do exposto nos papéis (3) (SILVA et al., 2014).

O analista primeiramente estabelece o objetivo, que será avaliação do consumo de energia incorporada e emissão de GHGs do berço ao fim-de-vida, e define o escopo que conduzirá a ACV (SILVA et al, 2014). Assim, procede-se à etapa de Análise de Inventário do Ciclo de Vida. O analista realiza a coleta de dados a partir dos projetos $2 \mathrm{D}$ (4). Estes dados são validados, relacionados por unidade de processo e agregados. A coleta de dados pode ser um processo que demanda muitos recursos. De acordo com a NBR ISO 14044 (ASSOCIAÇÃO BRASILEIRA DE NORMAS TÉCNICAS, 2009), a análise de inventários é um processo interativo, pois à medida que dados são coletados e se amplia o conhecimento sobre o sistema, novos requisitos ou limitações dos dados podem ser identificados, requerendo mudança nos procedimentos de coleta de dados, de modo que os objetivos do estudo possam ainda ser satisfeitos. A partir desta coleta, é realizada a avaliação de impacto do ciclo de vida (5), que tem como objetivo estudar a significância dos impactos ambientais potenciais, utilizando os resultados do ICV (ASSOCIAÇÃO BRASILEIRA DE NORMAS TÉCNICAS, 2009a).

Feito o procedimento anterior, procede-se à interpretação destes resultados, através de relatórios, caso se verifique a necessidade de revisão da meta ou escopo, a ACV volta ao início da análise, caso contrário é feita uma revisão crítica (6). Nesta, verifica-se a necessidade de revisão dos resultados, cálculos, interpretação ou relatório, caso haja a necessidade de revisão em qualquer um destes pontos, o processo retorna ao ponto referido, caso contrário o relatório final da ACV é emitido e passado ao cliente (7). Se a performance ambiental não for aceita, retorna ao arquiteto para revisão do projeto, caso contrário, o mesmo é procedido à execução (8) (ASSOCIAÇÃO BRASILEIRA DE NORMAS TÉCNICAS, 2009a).

Já no fluxo de trabalho apresentado na Figura 4, as metas e usos do BIM para ACV são determinadas logo no início do processo de projeto (1) e repassadas para os projetistas (CAMBIAGHI; AMA, 2012), que de acordo com a meta e o escopo anteriormente definidos elaboram os modelos (2) arquitetônico e urbano, modelo da construção, estrutural, hidrossanitário, elétrico, dentre outros (CIC, 2011). Logo após, o projeto BIM é passado para o especialista ACV (3), que extrai dos modelos e planilhas todos os dados necessários para a avaliação de energia e emissão de GHG incorporadas no ciclo de vida de edificações, sendo estes: os quantitativos de materiais de construção, distâncias médias de transportes de materiais, energia utilizada durante a etapa de construção e o consumo de energia (RAMESH; PRAKASH; SHUKLA, 2010). Os dados são validados, relacionados para unidade do processo e agregados (4).

Procede-se então à execução da avaliação do impacto do ciclo de vida, através da simulação no programa SimaPro (SILVA et al., 2014), gerando-se assim os indicadores ambientais (5), que são interpretados produzindo-se o relatório (ASSOCIAÇÃO BRASILEIRA DE NORMAS TÉCNICAS, 2009). Nesta etapa, caso haja a necessidade de revisão da meta ou escopo, o procedimento é retornado aos projetistas que vão adaptar os modelos de acordo com a revisão proposta. Caso contrário, o processo segue à revisão crítica (6), quando se verifica a necessidade de revisão dos resultados, cálculos, interpretação ou relatório, caso se verifique esta necessidade, o processo retorna ao ponto onde ela é necessária, caso contrário procede-se à execução do relatório final da ACV (ASSOCIAÇÃO BRASILEIRA DE NORMAS TÉCNICAS, 2009).

O relatório final da ACV é então repassado ao cliente (7). Se o relatório for aceito, procede-se ao desenvolvimento do projeto. Do contrário, retorna ao time de projeto, que novamente adapta o projeto até que seja alcançado o desempenho desejado (8).

\section{Impacto potencial de adoção de BIM na ACV}

Comparando-se os mapas de processo das Figuras 3 e 4, de avaliação de (energia e emissões GHG incorporadas no) ciclo de vida de edificações sem e com adoção de BIM respectivamente, pode-se especular médio impacto de implantação de BIM na ACV (Quadro 3).

\begin{tabular}{|c|c|c|c|c|c|}
\hline Processo & Impacto & Software & Impacto & $\begin{array}{l}\text { Informaç } \\
\text { ão }\end{array}$ & Impacto \\
\hline $\begin{array}{l}\text { Atividades } \\
\text { não sofrem } \\
\text { alterações }\end{array}$ & 1 & \multirow{2}{*}{$\begin{array}{c}\text { Usa } \\
\text { softwares } \\
\text { e estes } \\
\text { sofrem } \\
\text { alteraçõe } \\
\text { s }\end{array}$} & \multirow{2}{*}{2} & \multirow{2}{*}{$\begin{array}{c}\text { Era } \\
\text { parcialm } \\
\text { ente } \\
\text { analógica } \\
\text { /digital }\end{array}$} & \multirow{2}{*}{2} \\
\hline $\begin{array}{l}\text { Atividades } \\
\text { são } \\
\text { alteradas }\end{array}$ & 2 & & & & \\
\hline \multicolumn{6}{|c|}{ Impacto Total (soma): MÉDIO (5 ou 6 ) } \\
\hline
\end{tabular}

Fonte: BARROS (2016, p.88) 
Figura 3. Fluxo de trabalho de avaliação de (energia e emissões GHG incorporadas no) ciclo de vida de edificações

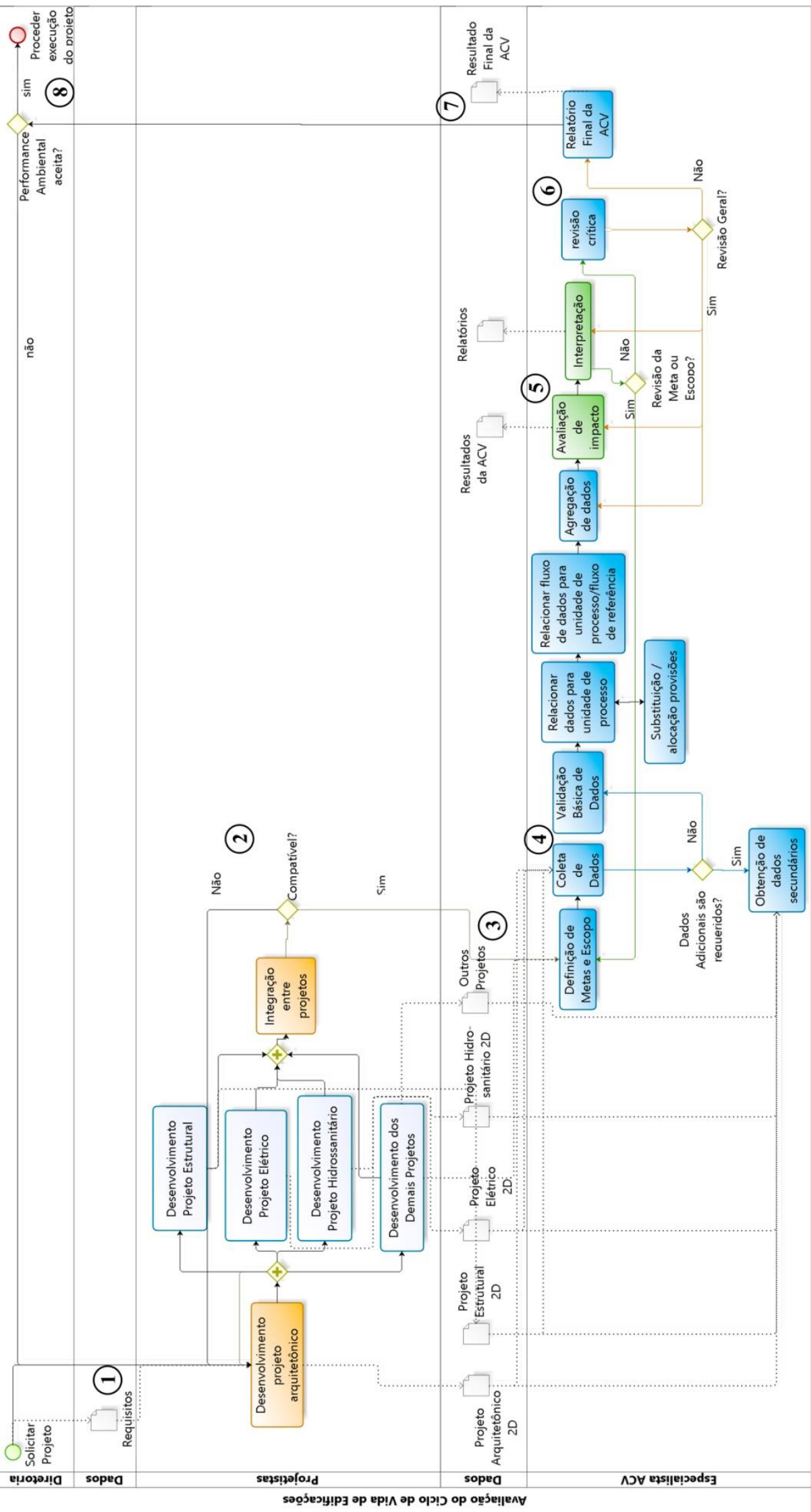

Fonte: Barros (2016, p.83) 
Figura 4. Fluxo de trabalho de avaliação de (energia e emissões GHG incorporadas no) ciclo de vida de edificações com adoção de BIM

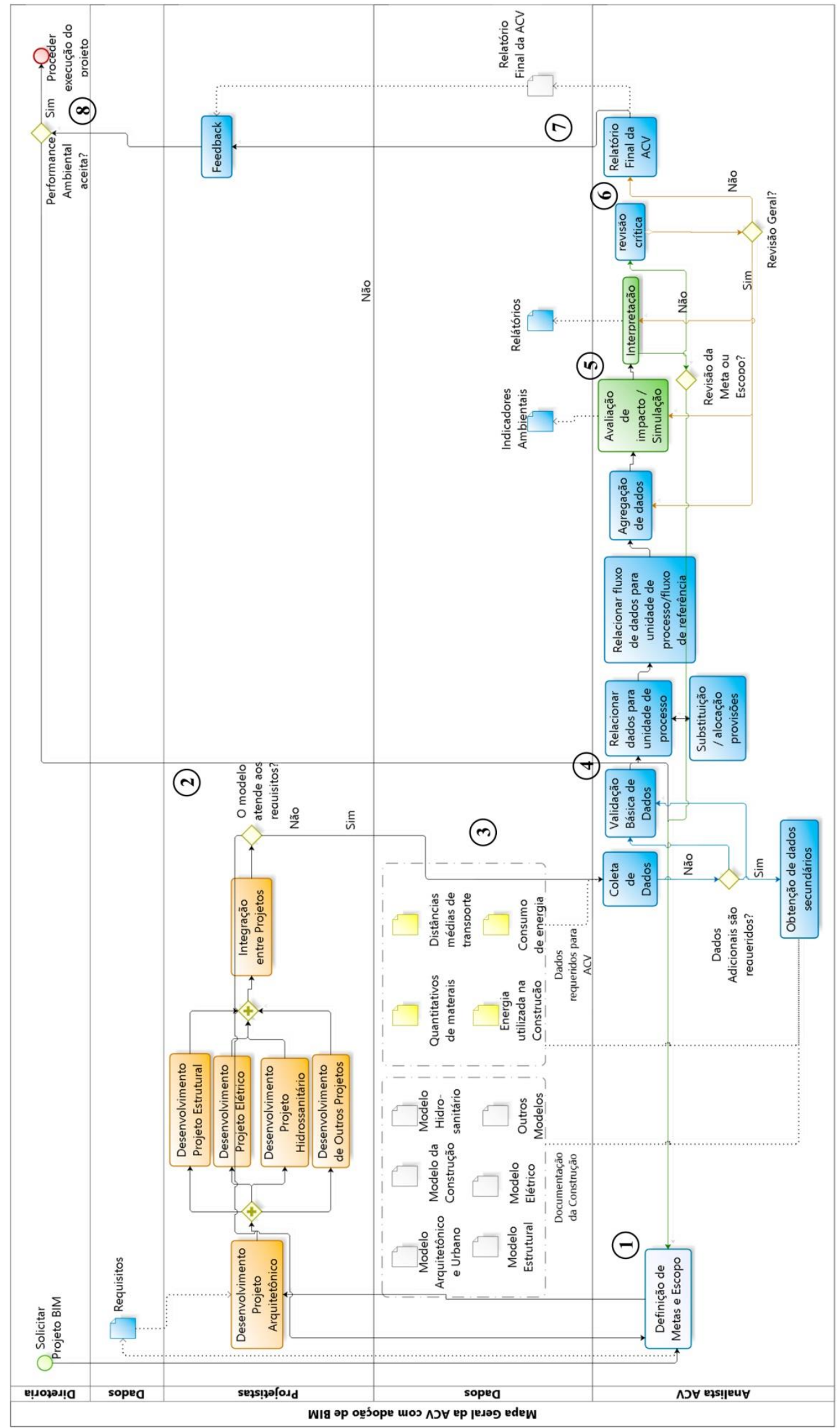

Fonte: Barros (2016, p.84) 
Em relação ao processo, o impacto é variado, uma vez que o reordenamento de atividades, através da definição do objetivo e escopo da ACV antes do desenvolvimento do projeto, pode ocorrer mesmo sem a adoção de BIM. Assim, caso a equipe não adote BIM, mas já realize a definição do objetivo e escopo da ACV a fim de nortear o projeto da edificação, o impacto da adoção de BIM é então menor.

Em relação à tecnologia associada, ocorre alteração com a adoção de BIM, no que tange ao programa utilizado para o desenvolvimento do projeto, alterando-se de sistemas CAD 2D para um software de modelagem BIM.

$\mathrm{E}$ as informações trocadas, que até então eram parcialmente analógicas/digitais (analógicas no que se refere aos projetos em papéis e, digitais no que se refere aos indicadores ambientais obtidos pelos softwares ACV) passaram a ser totalmente digitais.

\section{Discussão}

Esta pesquisa soma-se aos trabalhos que buscam integrar BIM e ACV, proporcionando uma contribuição teórica para o tema em questão e auxiliando no desenvolvimento de novas pesquisas nesta área.

A revisão sistemática da literatura desenvolvida revelou que do total de artigos encontrados, o método de extração automática de dados a partir do modelo BIM foi o mais abordado pelos artigos (25\%), seguido pelas pesquisas em interoperabilidade entre as ferramentas computacionais (21\%). Outros artigos trataram de desenvolver novos artefatos computacionais para integrar BIM e performance ambiental, através da otimização (8\%) e da programação (8\%). Esta revisão demonstra que o principal foco dos artigos atualmente publicados se encontra no campo da tecnologia, são raros os artigos publicados nos campos do processo e da política, revelando a grande necessidade de maiores estudos também nestas áreas.

Apesar de BIM mostrar-se uma ferramenta inovadora e que pode facilitar o processo de avaliação do ciclo de vida de edificações, todos os estudos realizados verificaram que a integração entre BIM e ACV, ou é inexistente, ou ainda apresenta muitos desafios. Isto revela que o tema é tão promissor quanto embrionário.

A análise comparativa entre os mapas de processos apresentados revelou impactos da adoção de BIM na avaliação de energia e carbono incorporados do ciclo de vida de edificações: no processo e nas informações trocadas, já os agentes envolvidos não sofreram alterações. A definição da meta e do escopo da ACV no início do processo, determina como o projeto será elaborado pela equipe, o que pode ser realizado mesmo sem a adoção de BIM. Já, as informações trocadas entre os agentes se alteram, da representação em 2D e em papéis, os projetos passam a ser modelos digitais, com informações em planilhas geradas automaticamente.

Em um processo linear tradicional de ACV, perde-se uma das principais vantagens do uso de BIM (iteração até chegar na solução otimizada) desde as etapas iniciais de projeto. Os indicadores ambientais só são obtidos ao final de todo o processo para uma solução definida. Isto faz com que alterações do projeto para melhorar o desempenho ambiental da edificação sejam limitadas.

Assim, verifica-se o potencial de melhorias no campo de processo e da tecnologia para otimização do desempenho ambiental da edificação, a fim de auxiliar o projetista na escolha de materiais de construção durante a etapa de projeto. A inserção de parâmetros que possibilitem o cálculo de energia e emissão de GHGs incorporadas no ciclo de vida de edificações, assim como qualquer outra informação ambiental, diretamente no modelo BIM, pode realmente transformar este processo e com impactos significativos no desempenho do ambiente construído, enquanto a desejada interoperabilidade completa entre softwares não é disponibilizada.

Deste modo, propôs-se a otimização do mapa de processo de avaliação de energia e emissão de GHG incorporada no ciclo de vida de edificação com adoção de BIM, apresentado na Figura 5. Primeiramente, o empreendedor solicita o projeto BIM, e passa os requisitos necessários para o analista, que define meta e o escopo da ACV (1). Logo após, os projetistas recebem os requisitos do empreendedor e os requisitos ambientais do analista ACV e procedem ao desenvolvimento do estudo preliminar (2).

Os dados referentes aos materiais de construção que possivelmente serão utilizados, são repassados aos fornecedores e ao especialista ACV. O primeiro coleta os dados dos materiais e fornece informações relativas ao transporte, e outras informações técnicas que são repassadas ao especialista ACV (3). O analista coleta os dados recebidos (4), valida-os, relaciona-os à unidade do processo e procede à análise de impactos (5), resultando nos indicadores ambientais por unidade funcional. Os indicadores são, então, repassados à equipe de projeto, junto com as informações relativas à distância de transporte, e procedem ao desenvolvimento dos projetos (6).

Os projetistas realizam as configurações necessárias no software BIM, incorporando informações ambientais nos componentes do modelo BIM, para o cálculo final dos indicadores ambientais (7). Caso o projeto não atenda aos requisitos (8), ele retorna à etapa anterior de projeto para modificações, caso contrário, os resultados são enviados ao especialista ACV para análise crítica e emissão do relatório final (9). Caso a performance ambiental seja aceita pelo empreendedor procede-se à execução do 
Figura 5. Fluxo de trabalho otimizado de avaliação de (energia e emissões GHG incorporadas no) ciclo de vida de edificações com adoção de BIM

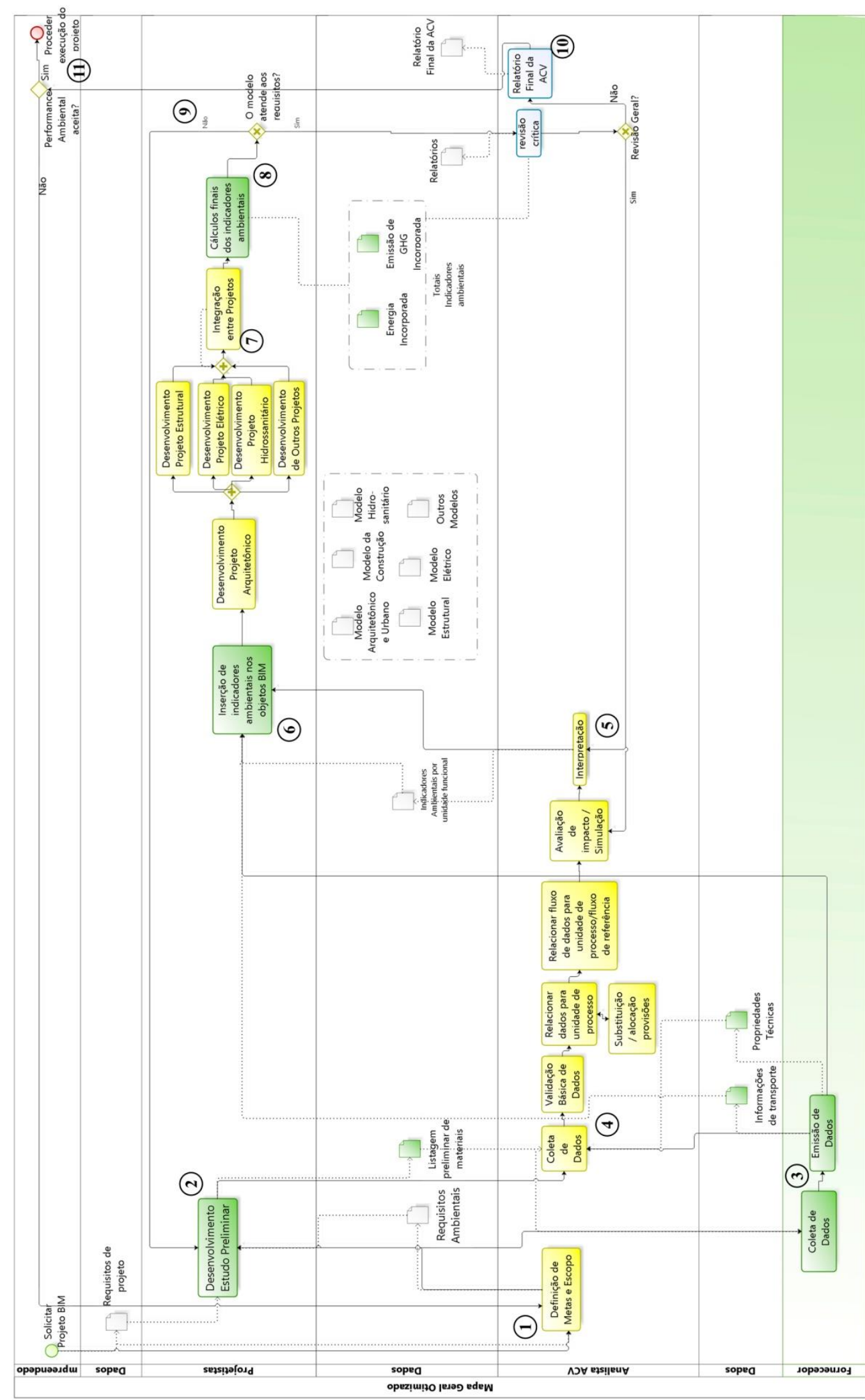

Fonte: $\operatorname{Barros}(2016, p .90)$ 
projeto (10), caso contrário, retorna novamente ao especialista ACV para adequações do objetivo e escopo.

\section{Conclusão}

A principal vantagem do uso de BIM na ACV é a sua capacidade de otimizar o processo, auxiliar a tomada de decisão durante toda a evolução do projeto e a convergência para uma solução otimizada. Particularmente em um projeto em desenvolvimento que tenha a interação dos agentes, o benefício de BIM+ACV pode ser ainda maior.

Na ACV tradicional, uma vez que o objetivo é definido, todos os quantitativos são extraídos manualmente e só então a ACV é processada. Se há mudanças na solução, a listagem de material deve ser reprocessada a cada vez. A execução dos cálculos referentes aos indicadores ambientais, dentro do próprio programa, pode fornecer ao projetista um feedback automático e fundamentar uma tomada de decisão mais robusta para edifícios com melhor desempenho ambiental. O ideal seria a interoperabilidade completa entre plataformas ACV e BIM, isto é: que o programa BIM permitisse exportação direta de informações para cálculo automático em softwares ACV.
Para tanto, é necessário o desenvolvimento de programas e/ou plug-ins que permitam essa integração. No estado de desenvolvimento atual, os inputs de parâmetros ambientais ainda precisam ser inseridos manualmente. No modo BIM+ACV, mesmo que a completa interoperabilidade entre softwares BIM e plataformas $\mathrm{ACV}$ não esteja disponível, quando o modelo de informação é preparado para fins de $\mathrm{ACV}$, o projetista pode solicitar que o especialista ACV calcule os impactos por unidade funcional de uma série de alternativas para uma determinada aplicação e inserir essas "propriedades" em um componente do projeto e ter o desempenho ambiental imediatamente recalculado dentro do próprio modelo. O fluxo de informação torna-se assim mais dinâmico

ACV é fundamental para alcançar as metas estabelecidas globalmente para diminuir os impactos dos edifícios. A utilização de tal procedimento na prática projetual é uma das principais peças que faltam para permitir a responsabilização ambiental rápida de decisões de projeto e para orientar a tomada de decisões na busca de soluções avançadas. BIM pode ajudar e deve ser explorado neste sentido.

\section{Agradecimentos}

Os autores agradecem o apoio financeiro concedido pela CAPES.

\section{Referências}

AL-GHAMDI, S.; BILEC, M. Green Building Rating Systems and Environmental Impacts of Energy Consumption from an International Perspective. In: ICSI 2014. [s.1.] Proceedings... American Society of Civil Engineers, 2014. p. 631-640.

ALWAN, Z.; JONES, P. The importance of embodied energy in carbon footprint assessment. Structural Survey, v. 32, n. 1, p. 4960, 2014.

ASSOCIAÇÃO BRASILEIRA DE NORMAS TÉCNICAS (ABNT). NBR ISO 14040: Gestão ambiental - Avaliação do ciclo de vida - Princípios e estrutura. Rio de Janeiro: ABNT, 2009.

ASSOCIAÇÃO BRASILEIRA DE NORMAS TÉCNICAS (ABNT). NBR ISO 14044: Gestão ambiental - Avaliação do ciclo de vida - Requisitos e orientações. Rio de Janeiro: ABNT, 2009.

BARROS, N.N. Impactos da adoção de BIM na avaliação de energia e emissões de GHG incorporadas no ciclo de vida de edificações. 2016. Dissertação (Mestrado em Arquitetura, Tecnologia e Cidade) - Universidade Estadual de Campinas, Campinas, 2016. Disponível em: http://www.bibliotecadigital.unicamp.br/document/?code=000974259

BASBAGILL, J.; FLAGER, F.; LEPECH, M.; FISCHER, M. Application of life-cycle assessment to early stage building design for reduced embodied environmental impacts. Building and Environment, v. 60, p. 81-92, 2013.

http://dx.doi.org/10.1016/j.buildenv.2012.11.009

BECERIK-GERBER, B.; KENSEK, K. Building Information Modeling in Architecture, Engineering, and Construction: Emerging Research Directions and Trends. Journal of Professional Issues in Engineering Education and Practice, v. 136, n. 3, p. 139147, 2010. http://dx.doi.org/10.1061/(ASCE)EI.1943-5541.0000023 
CAMBIAGHI, H.; AMA, R. Manual de Escopo de Projeto e Serviços de Arquitetura e Urbanismo. 2. ed. São Paulo: ASBEA, 2012. v. 1

CIC. Computer Integrated Construction Research Program, BIM Project Execution Planning Guide - Version 2.1. May, The Pennsylvania State University, University Park, PA, USA, 2011.

DAWOOD, S.; LORD, R.; DAWOOD, N. Development of a visual whole life-cycle energy assessment framework for built environment. In: WINTER SIMULATION CONFERENCE, 2009, Austin. Proceedings.... Austin: WSCF, p.2653-2663, 2009. http://dx.doi.org/10.1109/WSC.2009.5429263

DIAZ, J.; ANTON, L. Sustainable Construction Approach through Integration of LCA and BIM Tools. In: INTERNATIONAL CONFERENCE ON COMPUTING IN CIVIL AND BUILDING ENGINEERING, 2014, Orlando. Proceeding ... Orlando: ASCE, p. 283-290, 2014. http://dx.doi.org/10.1061/9780784413616.036

EASTMAN, C.; TEICHOLZ, P.; SACKS, R.; LISTON, K. Manual de BIM: um guia de modelagem da informação da construção para arquitetos, engenheiros, gerentes, construtores e incorporadores. Tradução: AYRES FILHO, C. G.; CÉSAR JÚNIOR, K. M.; FERREIRA, R.C.; FERREIRA, S.L.- Porto Alegre: Bookman, 2014.

IDDON, C. R.; FIRTH, S. K. Embodied and operational energy for new-build housing: A case study of construction methods in the UK. Energy and Buildings, v. 67, p. 479-488, dez. 2013. http://dx.doi.org/10.1016/j.enbuild.2013.08.041

IPCC, 2007: Climate Change 2007: Synthesis Report. Contribution of Working Groups I, II and III to the Fourth Assessment Report of the Intergovernmental Panel on Climate Change [Core Writing Team, Pachauri, R.K and Reisinger, A. (eds.)]. IPCC, Geneva, Switzerland, 104 pp.

JALAEI, F.; JRADE, A. Integrating BIM with Green Building Certification System, Energy Analysis, and Cost Estimating Tools to Conceptually Design Sustainable Buildings. In: CONSTRUCTION RESEARCH CONGRESS 2014. Proceedings...American Society of Civil Engineers, 2014. http://dx.doi.org/10.1061/9780784413517.015

JRADE, A.; JALAEI, F. Integrating building information modelling with sustainability to design building projects at the conceptual stage. Building Simulation, v. 6, n. 4, p. 429-444, 2013. http://dx.doi.org/10.1007/s12273-013-0120-0

KITCHENHAM, B.; CHARTERS, S. Guidelines for performing Systematic Literature Reviews in Software Engineering. UK, 2007.

KOWALTOWSKI, D.C.C.K.; CELANI, M.G.C.; MOREIRA, D.C.; PINA, S.A.M.; RUSCHEL, R.C.; SILVA, V.G.; LABAKI, L.C.; PETRECHE, J.R.D. 2006. Reflexão sobre metodologias de projeto arquitetônico. Ambiente Construído, 6 (2): 07-19. Disponível em: http://seer.ufrgs.br/ambienteconstruido/article/view/3683

MOREIRA, Lorena Claudia de Souza; RUSCHEL, Regina Coeli. Impacto da adoção de BIM em Facility Management: uma classificação. PARC Pesquisa em Arquitetura e Construção, Campinas, SP, v. 6, n. 4, p. 277-290, dez. 2015. ISSN 1980-6809. doi:http://dx.doi.org/10.20396/parc.v6i4.8634982.

OBJECT MANAGEMENT GROUP. Business Process Model and Notation (BPMN) - version 2.0. [s.1.] OMG, 2011.

PENG, C. Calculation of a building's life cycle carbon emissions based on Ecotect and building information modeling. Journal of Cleaner Production, v. 111, part 1, p. 453-465, jan. 2016. http://dx.doi.org/10.1016/j.jclepro.2015.08.078

RAMESH T.; PRAKASH, R.; SHUKLA, K.K. Life cycle energy analysis of buildings: An overview. Energy and Buildings, v. 42, n. 10, p. 1592-1600, 2010. http://dx.doi.org/10.1016/j.enbuild.2010.05.007

SHADRAM, F. et al. An Integrated BIM-Based Framework for the Energy Assessment of Building Upstream Flow. In: INTERNATIONAL CONFERENCE ON CONSTRUCTION AND REAL ESTATE MANAGEMENT, 2015, Lulea. Proceedings...Lulea: American Society of Civil Engineers, 2015. http://dx.doi.org/10.1061/9780784479377.013

SILVA, V.G.; SAADE, M.R.M. ; LIMA, B. W. F. ; MININEL, L. S. ; SILVA, M. G. . Life beyond operational stage: exploring lifecycle zero energy definitions. In: iSBE NET ZERO BUILT ENVIRONMENT 2014 SYMPOSIUM, 2014, Gainesville.

Proceedings... Gainesville: iiSBE, 2014. v. 1. p. 499-517. 
SUCCAR, B. Building information modelling framework: A research and delivery foundation for industry stakeholders. Automation in Construction, v. 18, n. 3, p. 357-375, 2009. http://dx.doi.org/10.1016/j.autcon.2008.10.003

WU, P.; XIA, B.; WANG, X. The contribution of ISO 14067 to the evolution of global greenhouse gas standards - A review. Renewable and Sustainable Energy Reviews, v. 47, p. 142-150, 2015. http://dx.doi.org/10.1016/j.rser.2015.02.055

\section{${ }^{1}$ Natália Nakamura Barros}

Arquiteta e Urbanista. Mestre em Arquitetura, Tecnologia e Cidade pela Faculdade de Engenharia Civil, Arquitetura e Urbanismo da UNICAMP. Endereço postal: Av. Ede, 550, São Paulo, SP, Brasil, 02203-001

\section{${ }^{2}$ Vanessa Gomes da Silva}

Arquiteta e Urbanista. Livre Docente na UNICAMP. Doutora e mestre em Engenharia Civil pela Escola Politécnica da USP. Endereço postal: Av. Albert Einstein, 951, Campinas, SP, Brasil, 13.083-852 